ich darf noch einmal meiner ganz besonderen Freude Ausdruck geben, daß Sie heute nun einmal bei mir zu Gaste sind; lassen Sie mich die Hoffnung daran knüpfen, daß Sie sich in Jena wohl fühlen mögen, und daß die Stadt Jena, deren Vorzüge Herr Oberbürgermeister $\mathrm{Fuchs}$ bereits rübmte, einen angenehmen und erfreulichen Eindruck bei Thnen hinterlassen möge, so daß Sie gerne auch später noch sich der diesmaligen Tagung in Jena erinnern werden. (Beifall.)

Vorsitzender: Ich nehme an, daß das Wort nicht mebr gewünscht wird, und danke für die freundlichen Worte der Begrüßung und Anerkennung und für das Interesse, das Sie unseren Bestrebungen durch Ihre Worte entgegengebracht haben. Sie werden uns Anlaß geben, auch auf der diesjährigen Tagung den Wünschen und Hoffnungsn gereeht zu werden, die an uns gestellt werden.

Wir gehen nunmehr zur Tagesordnung über, und ich gebe zunächst das Wort dem Herrn Ministerialrat Prof. Dr. Juckenack zu seinem Vortrag.

\title{
Über Ernährungsfragen vom Standpunkte der Wissenschaft, Wirtschaft und Gesetzgebnng.
}

\author{
Von \\ Prof. Dr. A. Juckenack in Berlin.
}

Trotzdem der Mensch von der Geburt bis zum Tode ununterbrochen auf Lebensmittel angewiesen ist, um leben, gedeihen und arbeiten zu können, trotzdem also die Lebensmittelfrage die wichtigste Existenzfrage aller Völker ist, mit deren Lösung schon im grauen Altertum das Nomandenleben, der Übergang zur Seßhaftigkeit in fruchtbaren Gegenden, Völkerwanderungen und Kriege zusammenhingen, ist seit der Entwickelung der Naturwissenschaften von diesen den Ernährungsfragen nicht immer die Beachtung geschenkt worden, die sie verdienen. Auch den Regierungen hatte ein weiter Blick für diese Fragen gefehlt. Sie hatten es nicht verstanden, die Naturwissenschaften rechtzeitig für die Lösung großer volkswirtschaftlicher Ernährungsprobleme zu interessieren; sie befabten sich vielmehr vorwiegend mit den jeweilig eine Rolle spielenden allgemeinen außen- und innenpolitischen Verhältnissen. Diese Tatsache trat plötzlich und mächtig in die Erscheinung, als England in den Weltkrieg eintrat und mit gewaltigen Mitteln sowie mit aller Energie systematisch dahin arbeitete, die Zufuhr von Lebensmitteln nach Deutschland abzuschneiden, ein Land, dessen eigene Landwirtschaft seine Bewohner nicht ausreichend ernähren konnte, das also schon unmittelbar nach .Kriegsbeginn sorgfältig darauf Bedacht nehmen mußte, mit den Erträgnissen seines Bodens so haushälterisch als nur denkbar zu wirtschaften. Damals erkannten alshald die einsichtigen Vertreter der Verwaltung und Volkswirtschaft, auf welch schwachen Füßen unsere Ernährungswissenschaft ruhte, wie mangelhaft sie zur Lösung gewaltiger Aufgaben der staatlichen Organisation ausreichte. So erklärt es sich ohne weiteres, daß damals sogar von Vertretern der Wissenschaft ganz unbrauchbare, wirtschaftlich vollständig undurchführbare Vorschläge gemacht wurden und zum Teil leider Anklang fanden, weil sie den Laien in der Verwaltung vorübergehend blenden konnten. Wir können es heute offen aussprechen, dalo bis zum Beginn des großen Weltkrieges in allen Kulturstaaten die Ernährungwissenschaft — vom Standpunkte der staatlichen Fürsorge für die allge- 
meine Volksernährung in wirtschaftlich schwierigen Zeiten betrachtet - vernachlässigt worden war. Eine Erklärung hierfür zu finden ist nicht schwer.

In den Kulturstaaten war mit der Zunahme des Wohlstandes und der Kultur allerdings das Interesse für Nahrungs- und Genußmittel gestiegen; küchentechnisch und fabrikmäßig war die Herstellung der Speisen immer mehr und mehr entwickelt worden; die Erfolge der Hygiene fanden Beachtung; die Ausdehnung des Verkehrs sorgte für Abwechslung und Vielseitigkeit; die Kunst der Zubereitung der Lebensmittel und der Art ihrer Darbietung hatte eine gewisse Höhe erreicht; aber diese Entwickelung vollzog sich unter Verhältnissen, unter denen man eine Lebensmittelnot nicht kannte, nicht ahnte, vielmehr infolge eines Uberflusses an Lebensmitteln ständig der Anreiz dazu wuchs, diesen fortgesetzt einen höheren Genußwert zu verleihen; denn selbst die breitesten Schichten des Volkes waren im allgemeinen in der Lage, sich ausreichende Mengen der wichtigsten Lebensmittel zu beschaffen; je nach der wirtschaftlichen Lage und den Bedürfnissen des einzelnen war lediglich die Auswahl der Nahrungs- und Genußmittel verschieden. Unter diesen Umständen konnten damals ernste Fragen der allgemeinen Volksernährung keine ausschlaggebende Bedeutung erlangen. Allerdings haben sich schon seit geraumer Zeit hervorragende Forscher mit der Frage beschäftigt, in welchem Umfange der Mensch je nach Alter, Geschlecht, Größe und Betätigung wichtige Nährstoffe wie Eiweißstoffe, Kohlenhydrate und Fette gebraucht; es wurde fortgesetzt versucht, über die chemische Zusammensetzung der verschiedenen Nährstoffe, Nahrungs- und Genußmittel, über ihr Verbalten bei der Zubereitung sowie Verarbeitung im menschlichen Körper usw. Aufschluß zu gewinnen; auch in gesundheitlicher Hinsicht ist das Verbalten zahlreicher Stoffe und Zubereitungen, die dazu bestimmt waren, vom Menschen genossen zu werden, erforscht worden; weiter hatte man ermittelt, wie viele Kalorien, d. h. welche Wärme-(Energie-) mengen, die bei der Oxydation der Lebensmittel im lebenden Körper auftreten, zum Unterhalt des menschlichen Körpers im allgemeinen erforderlich sind, und welche Bedeutung hierbei den wichtigsten Eiweißstoffen zukommt; alle diese Arbeiten hatten jedoch bis zum Kriegsausbruch streng genommen nur eine gewisse informatorische Bedeutung erlangt. Denn die Zahl der verschiedenen Eiweißstoffe, Fette und Kohlenbydrate in tierischen und pflanzlichen Nährstoffquellen ist außerordentlich grob; die chemische Konstitution zahlreicher wichtiger Näbrstoffgruppen ist bis beute noch unbekannt; ebensowenig wissen wir über sie vielfach heute noch in physiologischer Hinsicht $(z, B$, über die Bedeutung versehiedener pflanzlicher und tierischer stickstoffhaltiger Körper als Nahrungsmittel des Menschen); die Ausnutzungsversuche am Menschen wurden - vom Standpunkte der exakten Wissenschaft betrachtet - bisher noch recht summarisch, z. T. grob durchgeführt, indem man z. B. in den Stoffwechselprodukten des Menschen den gesamten Gehalt an Stickstoff, an in Äther löslichen Stoffen, die man Fette nannte, an solchen Mineralstoffen, die beim Veraschen hinterblieben, an sogenannter Cellulose, die aber solche im chemischen Sinne nicht war, usw. bestimmte. Man multiplizierte die analytisch ermittelte Stickstoffmenge mit 6,25, einem Faktor für gewisse Eiweißstoffe, die jedoch in ihrer chemischen Konstitution recht verschiedenartig waren, ganz abgesehen davon, daß dieser Faktor für zahlreiche Stickstoffverbindungen überhaupt nicht zutraf. Weiter war noch ziemlich unbekannt, welchen Einfluß der Gehalt der Nahrung an Kohlenhydraten und Fetten auf den Eiweißbedarf des Menschen hatte; die wirkliche Bedeutung der anorganischen Verbindungen konnte schon deswegen nicht erkannt werden, weil im allgemeinen die 
sogenannte Nährsalzfrage nach der Zusammensetzung von Aschen beurteilt wurde, die jedoch einen bedenkenfreien Rückschlu@ schon deswegen nicht zulassen kann, weil Calcium, Magnesium, Kalium, Natrium, Eisen usw. in den Aschen in ganz anderer Verbindung vorliegen müssen wie in den ursprünglichen Lebensmitteln, ganz abgesehen davon, da 3 in diesen je nach den Aufgaben der pflanzlichen und tierischen Zellen der Gehalt an mineralischen Stoffen, ihr Verbältnis zueinander und ihre Bindungsform recht verschieden sein dürften. Über die tatsächliche Bedeutung der verschiedenen Vitamine, zahlreicher Enzyme und vieler anderer Stoffe für den Lebensprozeß des Menschen war wenig bekannt. Mit den Ernährungsfragen hatte sich zudem vornehmlich die medizinische Wissenschaft befaßt, die sich vielfach hierfür allein für zuständig erachtete, der jedoch naturgemäß im allgemeinen eine tiefgehende Kenntnis der Chemie, der pflanzlichen Physiologie und anderer Naturwissenschaften fehlte und fehlen mußte, weil kein Mensch mehr in der Lage ist, alle naturwissenschaftlichen Disziplinen so gründlich zu beherrschen, wie es für die einwandfreie Klärung solcher Fragen erforderlich ist, die verschiedene Zweige der Naturwissenschaften eng berühren. Häufig - selbstverständlich nicht durchweg - glaubte der Mediziner, und das muß ganz offen ausgesprochen werden, daß der Chemiker für die Lösung von Fragen der hier in Rede stehenden Art überhaupt nicht in Frage komme, daß er sich mit ihnen nicht zu beschäftigen habe, weil er Laie sei und nur der Arzt in Ernährungsfragen sich ein wissenschaftliches Urteil zu bilden vermöge; es wirkte of recht eigenartig, wenn man erlebte, wie von ärztlicher Seite Männer wie Justus von Liebig abgetan wurden, wobei man vollständig übersah, daß man den chemischen Forscher, ebenso wie den medizinischen und jeden anderen Forscher, nur dann gerecht zu beurteilen vermag, wenn man sich vergegenwärtigt, wie der Stand der Wissenschaft, die allgemeine wissenschaftliche Erkenntnis zu jener Zeit war, als der zu kritisierende Forscher lebte. Was wußte z. B. zu Liebig's Zeiten der Mediziner von der exakten Chemie? Allerdings würdigte man späcer auch medizinischerseits die theoretischwissenschaftlichen Arbeiten eines Emil Fischer und anderer Geistesgrößen auf chemischem Gebiete, soweit sie sich nicht auf den Grenzgebieten zwischen Chemie

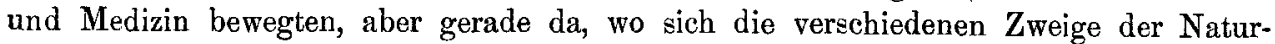
wissenschaften hätten begegnen müssen, entstanden leider die Konflikte. Es liegt mir selbstverständlich ganz fern, hiermit irgend einen Vorwurf gegen die Medizin erheben zu wollen; denn die geschilderten Verhältnisse waren nicht aus Vorurteil, sondern lediglich entwickelungsgeschichtlich aus dem Bestreben der einzelnen Disziplinen entstanden, je für sich ein möglichst weitgehendes Arbeitsgebiet abzugrenzen. Eine ähnliche Entwickelung ist überall da zu beobachten, wo sich in der Wissenschaft ein neues Fach herausbildet, das dann leider sehr leicht und bald die Fühlung oder Zusammenhänge mit den übrigen $Z$ weigen der Wissenschaft vorübergehend verliert. Auch Medizin und Chemie, selbst Physik sowie Pharmazie waren einst alle aufs engste miteinander verwachsen, als noch der einzelne Mensch alles zu übersehen vermochte, was auf diesen Gebieten wissenschafilich bekannt war.

Aber auch die Nahrungsmittelchemie entbehrte nicht der Einseitigkeit, auch bei ihr ist die bisherige Entwickelung die Folge ganz bestimmter Ursachen. Ich erinnere nur daran, daß in dem deutschen Strafgesetzbuch vom 1. Januar 1872 das Lebensmittelrecht, wenn man von den Paragraphen über Betrug sowie Vergiftung von Brunnen usw. absieht, noch ganz kümmerlich in $\S 367$ Ziffer 7 berücksichtigt worden ist. Denn hier wird mit Geldstrafe bis zu $1 \tilde{a} 0 \mathrm{Mk}$. oder entsprechender Haftstrafe bestraft, 
wer verfälschte oder verdorbene Eßwaren oder Getränke, insbesondere trichinenhaltiges Fleisch feilhält oder verkauft. Zweifellos lag damals noch kein zwingendes Bedürfnis für weitergehende Bestimmungen vor. Als aber nach dem deutsch-französischem Kriege in den sogenannten Gründerjabren unter dem Einfluß der Entwickelung der Naturwissenschaften und der Technik die Mißstände im Verkehr mit Lebensmitteln immer gemeingefährlicher wurden, schuf man ein besonderes Nahrungsmittelrecht, das immer weiter ausgebaut wurde, und zu dessen Durehführung man besondere Sachverständige heranbilden muPte. So entstand der hygienisch orientierte deutsche Nabrungsmittelchemiker, dessen Aufgabe vornehmlich die war, bei der Überwachung des Verkehrs mit Lebensmitteln den Aufsichtsbehörden wissenschaftlich und technisch einwandfreie Unterlagen für die Durchführung der Lebensmittelgesetze zu geben. Da diese Gesetze einen hygienischen Charakter haben, erklärt es sich, daß der medizinisch vorgebildete Hygieniker zunächst in der Nahrungsmittelchemie lediglich eine Hilfswissenschaft der Hygiene erblickte. Die Entwickelung der Verhältnisse auf dem Lebensmittelmarkt infolge der damals ungeahnten Entwickelung der Naturwissenschaften, der Technik, des Verkehrs und der Kultur hat jedoch bald dahin geführt, daß die Mißstände rein gesundheitlicher Art allmählich gegenüber den Mißständen wirtschaftlicher Art - auf dem Gebiete der Verfälschung und irreführenden Nachmachung von Lebensmitteln — nur noch eine untergeordnete Bedeutung hatten, daß mithin der Schwerpunkt der Nahrungsmittelkontrolle auf dem Gebiete des Lebensmittelchemikers, aber nicht des medizinisch vorgebildeten Hygienikers lag. Die wissenschaftliche Betätigung des Nahrungsmittelchemikers war in den wenigen Jahrzehnten seiner Existenz eine außerordentlich umfangreiche; der junge Stand setzte seine ganzen frischen Kräfte für seine Entwickelung ein; es entstand in der kurzen Zeit die umfangreiche nahrungsmittelchemische Literatur, die für den deutschen Lebensmittelchemiker zweifellos ein glänzendes Zeugnis ablegt. Sieht man diese Literatur durch, so erkennt man ohne weiteres, daß ihr Schwerpunkt in der Lösung der Aufgaben liegt, die dem Nahrungsmittelchemiker seinerzeit gestellt worden waren und die z. B. auch in der Prüfungsordnung für Nahrungsmittelchemiker zum Ausdruck kommen. Hier wie in der medizinischen Wissenschaft lag kein zwingender Anlab dazu vor, sich allgemeinen großen Ernährungsproblemen mit besonderer Energie zu widmen. So erklärt es sich ungezwungen, daßo diese Fragen auch von dem Nabrungsmittelchemiker stiefmütterlich behandelt worden sind, obwohl nicht zu verkennen ist, dab auch $\in \mathrm{r}$, ebenso wie der Mediziner, für die Lösung von Ernährungsfragen stets wichtige Beiträge geliefert hat.

Die Verhältnisse haben sich seitdem, wie ich eingangs meiner Ausführungen darlegte, gründlich geändert. Der Nahrungsmittelchemiker wird sich künftig neben seinen bisherigen Hauptaufgaben auch den allgemeinen Ernährungsfragen eingehend zu widmen haben, also diese, soweit sie chemischer Art sind, nicht lediglich der allgemeinen Chemie überlassen dürfen, die zudem und mit Recht rein theoretisch-wissenschaftliche Ziele bevorzugt.

Im Hinblick darauf, daß wir jetzt nach dem unglücklichen Ausgange des Weltkrieges in einer Zeit größter wirtschaftlicher Not leben, und daß die dem Deutsehen Reiche durch seine früheren Gegner bis in die neuste Zeit auferlegten Lasten diese wirtschaftliche Not lange Zeit anbalten lassen werden, ist es im Interesse des deutschen Volkes dringend erforderlich, dem Problem der Volksernährung nunmehr allseits die gröbte Beachtung zu schenken. In richtiger Erkenntnis der Tatsache, daß dieses 
große Problem in absehbarer Zeit nur durch intensives Zusammenwirken der Vertreter aller beteiligten Zweige der Naturwissenschaft eine wirklich ersprießliche Förderung erfahren kann, daß also bis auf weiteres die wissenschaftlichen Sonderinteressen zurückgestellt und vor allem die Vorurteile der einzelnen Zweige der Wissenschaft. gegen einander schwinden und alle Kreise zu gemeinsamer vorurteilsloser Arbeit zusammengefaßt werden müssen, hat in neuester Zeit der Reichsminister für Ernährung und Landwirtschaft Herr Dr. Hermes einen „Beirat zur Förderung ernährungswissenschaftlicher Forschungstätigkeit" (jetzt „Reichsausschuß für Ernährungsforschung") berufen. Er hat sich hierbei von der Absicht leiten lassen, mit allen beteiligten Kreisen enge Fühlung zu nehmen, um einerseits die $W$ issenschaft darüber zu unterrichten, welche Fragen die Reichsregierung vom Standpunkte der Volkswirtschaft besonders interessieren, und um andererseits aus den Kreisen aller beteiligten Zweige der Wissenschaft fortgesetzt Anregungen für die Volkswirtschaft entgegennehmen zu können. Daher finden wir in diesem Beirat z. B. die organische, anorganische und physikalische Chemie, die Ernährungsphysiologie, die Pflanzenphysiologie, die Vererbungslehre, die Nabrungsmittelchemie, die Agriculturchemie, die Bakteriologie, die Nahrungsmittelhygiene und die Ernährungsstatistik vertreten. Die Aufgaben, die dem Herrn Minister für den Beirat vorschweben, sind etwa in folgende Fragen zusammenzufassen:

1. Wie lassen sich die zur Verfügung stehenden Rohstoffe vom Standpunkte der Wissenschaft vorteilhafter als bisher ausnutzen?

2. Wie lassen sich die bisherigen Verfahren zur Verarbeitung der Rohstoffe auf Lebensmittel im Interesse einer bestmöglichen Ernährung der Bevölkerung vom Standpunkte der Wissenschaft zweckmäßiger gestalten?

3. Wie ist es der Bevölkerung möglich, die zur Zeit zur Verfügung stehenden Lebensmittel nicht nur möglichst schmackhaft, sondern zugleich auch so zuzubereiten, daß sie soweit als nur möglich und so zweckmäßig als nur möglich vom Körper ausgenutzt werden?

4. Können neue Nährstoffquellen für den Menschen erschlossen werden?

Der Herr Reichsminister für Ernährung und Landwirtschaft beabsichtigt, einschlägige Forschungen finanziell zu fördern, jedoch stehen ihm hierfür bisher nur geringe Mittel zur Verfügung. Infolgedessen und vor allem auch aus dem Grunde, um ein gemeinsames Zusammenarbeiten von Vertretern der verschiedenen wissenschaftlichen Kreise zu ermöglichen, konnte bisher die Zahl der Mitglieder des Beirats, unter denen sich auch Angehörige unseres Vereins befinden, nur eine beschränkte sein. Es ist im öffentlichen Interesse lebhaft $z u$ begrüßen, daß der den Herrn Minister leitende Gedanke auf fruchtbaren Boden gefallen ist, daß also die bisher berufenen Vertreter der verschiedenen Zweige der Wissenschaft sich vorurteilsfrei zu gemeinsamer Arbeit zur Verfügung stellten. Hiermit sind die Scheidewände, die früher aus den zuvor angegebenen Gründen vielfach künstlich zwischen den einzelnen Disziplinen errichtet worden waren, gefallen, und es wird jeder Forscher gern bestrebt sein, da zu helfen, wo er der guten Sache zu dienen vermag. Rein sachlich betrachtet sollte es zudem selbstverständlich sein, daß jeder Forscher nur auf dem Gebiete intensiv tätig ist, das er beherrscht, also eine gemeinsame Arbeit anstrebt, sobald die Lösung bestimmter Fragen die Kenntnisse melrerer wissenschaftlicher Disziplinen zur Voraussetzung hat. Wie dringend notwendig oft ein gemeinsames Arbeiten ist, mag nur an der Hand einiger Beispiele erläutert werden. 
Es ist unmöglich, die Min eralst of ffrage (Nährsalzfrage), also die Frage der Bedeutung der Mineralstoffe für den Lebensprozeb des Menschen sowie auch der Tiere und der Pflanzen, lediglich mit der Kenntnissen des Arztes, Tierarztes, Botanikers oder Chemikers lösen zu wollen. Um der Lösung der Frage ein festes Fundament zu geben, ist die Mitwirkung des anorganischen Chemikers unbedingt erforderlich. Wahrscheinlich mub hierbei auch noch der organische und physikalische Chemiker einsetzen. - Aus hoch interessanten physiologisehen Arbeiten der neueren Zeit wissen wir, welche Bedeutung die Vita mine, gewisse Ergänzungsstoffe der Nahrung, haben; wir wissen aus physiologischen Versuchen, in welchen Lebensmitteln Vitamine eine Rolle spielen und welche Bedeutung daher gewisse Tebensmittel bei der allgemeinen Zusammensetzung der Kost haben; wir wissen weiter, daß es verschiedene Vitamine gibt, deren Fehlen in der Nahrung verschiedenartige Krankheiten zur Folge haben kann, daß z. B. antiskorbutisches Vitamin im Citropen- und Orangensaft, in Tomaten, Äpfeln und anderen Lebensmitteln im Gegensatz zu den Vitaminen, die bei Rachitis, Nervenstörungen, Degenerationserscheinungen eine Rolle spielen, gegen Erhitzung empfindlich ist, aber wir wissen leider auch weiter, daß über diese Körper in chemjscher Hinsicht bisher nichts bekannt ist, ja wir kennen noch nicht einmal den Einfluß, den die verschiedenen Verfahren der Herstellung von Lebensmitteln und der Zubereitung von Speisen auf die darin enthaltenden Vitamine haben. - Ähnlich so liegt zum größten Teil auch noch das Gebiet der Enzyme im Dunkeln, dessen Erforschung unvorhersebbare wirtschaftliche und ernährungspbysiologische Folgen haben kann. - Aber selbst da, wo die exakte Naturwissenschaft bisher schon viel weiter vorgeschritten ist, gibt es in chemischer und physiologischer Hinsicht noch außerordentlich viel zu erforschen. Ich brauche nach dieser Richtung nur daran zu erinnern, wie wenig bisher das Gebiet der EiweiBstoffe exakt erforscht ist, in welch ungeheurem Umfange theoretisch die Möglichkeit der Existenz verschiedenartiger Eiweißstoffe besteht, und wie verschieden daher die Rolle sein kann, die dieses Heer von Eiweißstoffen und verwandten Stickstoffverbindungen in ernährungsphysiologischer Hinsicht spielen. - Selbst auf dem Gebiete der Fettchemie wissen wir, daß die genaue Zusammensetzung aller in den verschiedenen für die Ernährung in Betracht kommenden Fetten enthaltenen Glyceride bei weitem noch nicht bekannt ist. Interessant war es, als es in neuerer Zeit gelang, aus Koblenwasserstoffen Fettsäuren herzustellen. Aber selbst wenn auf diesem Gebiete die Chemie wesentliche Fortschritte machen und es ihr eines Tages gelingen sollte, in beliebigem Umfange und zu ersehwinglichen Preisen Fettsäuren chemisch zu gewinnen, so ist dann sorgfältig die Frage zu prüfen, ob diese Körper und ihre Ester nicht nur für technische $Z$ wecke (z. B. bei der Seifenfabrikation), sondern auch für die menschliche Ernährung verwertbar sind.

Der Beirat zur Förderung ernährungswissenschaftlicher Forschungstätigkeit beim Reichsernährungsministerium hat sich aber auch noch nach anderer Richtung Aufgaben gestellt, die auf dem Gebiete der Erzeugung von Lebensmitteln liegen. Z. B. soll auch geprüft werden, wie durch systematische Erdan aly se der Körnerertrag unseres Bodens erheblich gesteigert werden kann. Im Zusammenhange hiermit wird weiter die Bedeutung versehiedener Phosphorsäurefragen für die Mehrung der Ernte erforscht werden, nachdem in den letzten Jahren die Frage der Versorgung Deutschlands mit stickstoffhaltigen Düngemitteln gelöst ist. - Nachdem es auf ganz einfachem Wege gelungen ist (nach dem Beckmann'schen Verfahren), den Futterwert des Strohes durch Beseitigung von holzigen Substanzen um ein Vielfaches zu erhöhen, 
sind Versuche in Aussicht genommen, auf biologischem Wege die Cellulose aufzuschließen. Auch auf pflanzenphysiologischem Gebiete gibt es noch sehr interessante und wichtige Fragen - z. B. hinsichtlich der Aufspeicherung der Reservestoffe im reifen Samen der Kulturpflanzen - zu lösen. - Weiter interessiert z. B. die Frage des Anbaues der Sojabohne und der ErdnuB. Es würde viel zu weit führen, im Rahmen dieses Vortrages eingehend die Probleme zu erörtern, die für die Hebung der Volksernährung nach der angegebenen Richtung unter den gegenwärtigen Verhältnissen in Betracht kommen. Als Anlage werde ich im Druck dem Vortrage die Denkschrift beifügen, die der Herr Ernährungsminister in bezug auf den mehrerörterten Beirat und zwar zur Begründung seiner finanziellen Forderungen dem Reichstage vorgelegt bat.

Um die Nahrungsmittelchemiker weitestgehend zur Lösung der zahlreichen volkswirtschaftlichen Aufgaben heranzuziehen und sie insbesondere auch laufend darüber informieren zu können, welche Probleme jeweilig im Vordergrunde des öffentlichen Interesses stehen, dürfte es sich empfehlen, den Herrn Ernährungsminister zu bitten in seinen Beirat auch den Vorsitzenden unseres Vereins zu berufen, da der Verein bereits seit seiner Gründung satzungsmäßig die wissenschaftliche Forschung auf dem Gebiete der Lebensmittel energisch gefördert habe; allerdings habe sich die wissenschaftliche Tätigkeit seiner Mitglieder früher in der Hauptsache im Rahmen der allgemeinen Lebensmittelhygiene bewegt, weil es damals vornehmlich darauf ankam, die deutsche Lebensmittelgesetzgebung im Interesse der Volksgesundheit sowie zum Schutze der Bevölkerung gegen Ausbeutung durch unredliche Lebensmittelhersteller und -Händler möglichst zweckmäßig auszugestalten, um den mit der Überwachung des Verkehrs mit Lebensmitteln betrauten Behörden das erforderliche Rüstzeug zu liefern; daneben seien aber seit jeher von uns auch solche Fragen, die lediglich der allgemeinen Erforschung der Zusammensetzung und Verwertung der Lebensmittel dienen, behandelt worden; als im Laufe des Krieges eine tiefgreifende Änderung der früheren wirtschaftlichen Verhältnisse einsetzte, seì das Gebiet der allgemeinen Nahrungsmittelchemie auch von Mitgliedern unseres Vereins mehr als zuvor gepflegt worden, zumal damals amtlich alle Kräfte aufgefordert wurden, zu versuchen, dabei mitzuwirken, der Ernährungsschwierigkeiten Herr zu werden; unser Verein glaube daher mit Hilfe zahlreicher Mitglieder in der Lage zu sein, im Rahmen der vom Reichsernährungsministerium für die Aufgaben seines wissenschaftlichen Beirats gezogenen Grenzen wertvolle Beiträge in absehbarer Zeit liefern zu können; naturgemäß könne der Beirat nicht zu groß sein, wir möchten es aber für zweekmäßig erachten, jedenfalls nocb den Vorsitzenden unseres Vereins in ihn zu berufen, damit er in der Lage ist, die bei uns in Betracht kommenden Kräfte über die jeweiligen Fragen zu orientieren und zur Mitarbeit heranzuziehen.

Die chemische Großindustrie hat weit früher als die Regierung die Bedeutung der finanziellen Förderung der Wissenschaft erkannt. Denn es ist nicht zu verkennen, daß die schnelle Entwickelung und die großen Erfolge unserer Industrie zum Teil darauf zurückzuführen sind, daß von ihr fortgesetzt große Mittel für Forschungsarbeiten zur Verfügung gestellt wurden, und daß dadurch die Forscher ein Interesse daran bekamen, auf wirtschaftlichem Gebiete liegende wissenschaftliche Fragen zu lösen. Infolgedessen sollte die Reichsregierung nunmehr in der Zeit der Not ebenfalls versuchen, die Wissensehaft weitestgehend für ihre Aufgaben zu interessieren und zu dem $\mathrm{Zweck}$ Mittel zu beschaffen, um Forschungsarbeiten fördern zu können, 
was heute schon deswegen viel wichtiger als früher ist, weil alle in Betracht kommenden wissenschaftlichen Institute sich unter dem Druck der wirtschaftlichen Verhältnisse in großer Not befinden.

Die Nahrungsmittelchemiker haben sich übrigens schon einrnal sehr schnell in ihrer Tätigkeit umstellen müssen, und sie haben damals bewiesen, daß sie dies können, sobald ihnen besondere Aufgaben gestellt werden. Ich denke bierbei an die Zeit, als während des Krieges die Ersatzmittel für Lebensmittel und Gebrauchsgegenstände aller Art wie Pilze aus der Erde emporsproßten, und infolgedessen fortgesetzt neue wissenschaftliche Verfahren ausgearbeitet werden mußten, um den Verkehr mit Ersatzmitteln so zu regeln, daß er halbwegs erträglich wurde, und zwar in einer Zeit, in der wir weitestgehend auf Ersatzmittel angewiesen waren.

Der Nahrungsmittelchemiker wird sich demnächst aber nicht nur nach der zuvor geschilderten wissenschaftlichen Richtung, sondern auch noch anderweitig umzustellen baben. Denn wir stehen nunmehr unmittelbar vor einer grundlegenden Änderung unserer bisherigen Lebensmittelgesetzgebung, einer Frage, mit der wir uns vorbereitend bereits auf der vorjährigen Hauptversammlung unseres Vereins eingehend beschäftigt haben. Ich hatte gehofft, die Reichsregierung würde schon im Sommer d. Js. einen vorläufigen Gesetzentwurf der öffentlichen Kritik unterbreiten, und wir würden daher heute in der Lage sein, zu ihm vom Standpunkte unserer reichen, in Wissenschaft und Praxis gesammelten Erfahrungen gutachtlich Stellung zu nehmen. Dies ist leider nicht der Fall.

Vor wenigen Wochen (am 22. August 1921) batte ich anläßlich der Reichsausstellung für Kolonialwaren und Lebensmittel in Frankfurt (Main) Gelegenheit, auf der Hauptversammlung des Reichsverbandes Deutscher Kolonialwaren- und Lebensmittelhändler im Rahmen eines einstündigen Vortrages, der inzwischen im Verlage von Julius Springer in Berlin erschienen ist, in Form einer Skizze die Entstehung, Entwickelung und künftigen Aufgaben der deutschen Lebensmittelgesetzgebung zu schildern und zwar von ihren Uranfängen auf deutschem Boden an. Um dieselbe Zeit erhielt die Nürnberger Handelskammer vom bayerischen Handelsministerium den im Reichsgesundheitsamte ausgearbeiteten Entwurf für ein neues Gesetz über den Verkehr mit Lebensmitteln und anderen Bedarfsgegenständen. Infolgedessen ersuchte die Handelskammer den Bund Deutscher Nahrungsmittelfabrikanten und -Händler e. V. um gutachtliche Stellungnahme, der daraufhin seinen Gutachterbeirat sowie Vertreter der ihm angeschlossenen Fachverbände zu einer Sitzung einberief, die am 28. August d. Js. im großen Saal der Handelskammer zu Nürnberg unter Vorsitz von Herrn Fabrikbesitzer August Ertheiler stattfand. Da ich auf der vorjährigen Hauptversammlung mit der Vertretung unseres Vereins im Bunde Deutscher Nahrungsmittelfabrikanten und -Händler betraut worden war, wohnte ich der Versammlung bei und übernahm zugleich auf Wunsch ihres Vorsitzenden unter Berücksichtigung des Gesetzentwurfes das einleitende Referat zur Reform der Lebensmittelgesetzgebung. Die Verhandlungen verliefen durchaus sachlich und sehr anregend. $\mathrm{Zu}$ berücksichtigen ist, daß bisher ein formeller Gesetzentwurf der Reichsregierung noch nicht vorliegt, also noch nicht feststeht, wie sich die Reichsministerien sowie die Regierungen der Länder zu dem im Reichsgesundheitsamte ausgearbeiteten vorläufigen Entwurf stellen werden. Herr Ertheiler und ich haben jedoch Gelegenheit genommen, den Herrn Referenten des Reichsministeriums des Innern gelegentlich einer gemeinsamen Besprechung zu bitten, seinen Einfluß 
dahin geltend zu machen, daß, sobald der Regierungsentwurf vorliegt, dieser zunächst der öffentlichen Kritik unterbreitet werden möge, damit alle beteiligten Kreise in die Lage kommen, ihre Wünsche schon so rechtzeitig geltend zu machen, daß sie bei der Bearbeitung des endgültigen, für den Reichsrat und den Reichstag bestimmten Entwurfes soweit als angängig berücksichtigt werden können. Es ist damit zu rechnen, daß dieser Anregung stattgegeben wird, und infolgedessen werden wir uns auch darüber schlüssig machen müssen, wie wir unsererseits der Reichsregierung gegenüber demnächst zu ibrem vorläufigen Entwurf am besten Stellung nehmen können. Zu dem Zweck werden wir aus den Mitgliedern unseres Ausschusses und deren Vertretern eine besondere Kommission für das neue Nahrungsmittelgesetz bilden müssen, da der Zusammentritt des gesamten Ausschusses zu große Kosten verursacht (schätzungsweise etwa 12-13000 Mk.), und die Angelegenheit nicht bis zur nächsten Hauptversammlung vertagt werden kann, wenn diese nicht schon im kommenden Winter stattfinden soll, was ich aber nicht für zweckmäßig erachten würde, weil für die folgende Zeit noch wichtige Aufgaben bevorstehen. Denn es ist damit zu rechnen, daß im unmittelbaren Anschluß an das neue Nahrungsmittelgesetz die hierzu hinsichtlich der verschiedenen Lebensmittel zu erlassenden eingehenden Ausführungsbestimmungen sorgfältig beraten werden müssen, und es ist nicht zu verkennen, daß diese Ausführungsbestimmungen für die künftige Handhabung der Nahrungsmittelkontrolle von allergrößter Bedeutung sein werden, während das nene allgemeine Gesetz nur den Charakter eines Mantelgesetzes haben und somit gewissermaßen das Fundament der künftigen Lebensmittelgesetzgebung bilden wird.

Der bisher vorliegende Gesetzentwurf hat den Charakter eines derartigen Mantelgesetzes, indem er weitestgehend zwecks tunlichster Vermeidung von Sondergesetzen ein Verordnungsrecht des Reichsrates sowie weiter vorsieht, daß dessen Verordnungen gewissermaßen integrierende Bestandteile des neuen Gesetzes werden. Hier lehnt sich der Entwurf an das Ergebnis von Beratungen an, die am 27. März 1911 im Reichsgesundheitsrat mit Vertretern des Deutschen Landwirtschaftsrats, des Deutschen Handelstages, des Bundes der Industriellen, des Bundes Deutscher Nahrungsmittel-Fabrikanten und -Händler, des Verbandes Deutscher Großhändler der Nahrungsmittel- und verwandten Branchen sowie der Zentralvereinigung deutscher Vereine für Handel und Gewerbe stattgefunden haben, und bei denen die beteiligten Berufskreise einmü tig der Auffassung waren, daß nur durch rechtsverbindliche Festsetzungen über die Beschaffenheit und Beurteilung der einzelnen Lebensmittel die unleugbar vorhandenen Mißstände beseitigt werden können.

Der bisherige vorläufige Gesetzentwurf trägt die Überschrift: „Gesetz über den Verkehr mit Lebensmitteln und anderen Bedarfsgegenständen.“ Auf ihn näher öffentlich einzugehen, muß ich mir zur Zeit versagen, da eine amtliche Bekanntgabe des Entwurfs in der Presse bisher nicht erfolgt ist. Ich werde Ihnen jedoch vertraulich den wesentlichsten Inhalt mitteilen, zumal hiergegen schon deswegen keine Bedenken bestehen, weil der Entwurf zur Zeit auf amtliche Anregung hin in den wichtigsten wirtschaftlichen Verbänden beraten wird, und ich derartigen Erörterungen als Vertreter unseres Vereins beigewohnt habe. [Im Anschlub hieran wurde der vertrauliche Bericht erstattet.]

Der Bund Deutscher Nahrungsmittel-Fabrikanten und -Händler hat sich bisher zunächst mit der grundsätzlichen Frage beschäftigt, ob sich Handel und Industrie damit einverstanden erklären können, daß künftig von Sondergesetzen abgesehen und 
der Verkehr mit Lebensmitteln auf Grund eines allgemeinen Lebensmittelgesetzes durch Reichsratsverordnungen geregelt wird, um die Gesetzgebung beweglicher als bisher durch Erlaß von Sondergesetzen zu gestalten. Diese Frage ist nach eingehenden Beratungen von ihm wiederum (wie am 27. März 1911) bejaht worden. Auch von unserem Standpunkte aus kann es nicht zweifelhaft sein, daß diese Regelung die zweckmäßigste ist.

Die zweite, den Bund Deutscher Nahrungsmittel-Fabrikanten und -Händler besonders interessierende Frage ist die: Wie ist am zweckmäßigsten ein Gutachterausschuf von Handel, Industrie und Verbrauchern zu bilden, in dem die widerstreitenden Interessen der beteiligten Kreise zunächst gegeneinander abgewogen werden, um eine einheitliche Stellungnabme des Lebensmittelverkehrs zu gewährleisten und insbesondere zu verhüten, daß künftig bei den Beratungen im Reichsgesundheitsrat usw. die verschiedenen Interessentengruppen Erörterungen dadurch erschweren, daß sie sich gegenseitig bekämpfen, statt auf ein gemeinsames Ziel hinzusteuern? Denn jeder von uns, der im Laufe der Zeit in Reichsbehörden Verhandlungen über derartige Fragen auf dem Gebiete des Verkehrs mit Lebensmitteln und Gebrauchsgegenständen beigewohnt hat, weiß, daß man praktisch kaum weiter kommt, wenn sich die versehiedenen Interessentengruppen gegenseitig bekämpfen. Ich erinnere nach dieser Richtung z. B. nur an die vielen Verhandlungen über den Verkehr mit Ersatzlebensmitteln und an die Beratungen, betr. die Festsetzungen über den Verkehr mit Essig, Essigessenz u. dergl. Infolgedessen hat der Bund Deutscher Nahrungsmittel-Fabrikanten und -Händler beschlossen, einen Gutachterbeirat aus Vertretern der verschiedenen Verkehrskreise unter Zuziehung eines Vertreters des Vereins Deutscher Nahrungsmittelchemiker zu bilden, der zum Teil aus ständigen Mitgliedern zur Sicherung der Kontinuität, d. h. des ununterbrochenen Zusammenhanges zwischen allen Verkehrskreisen auch den Behörden gegenüber sowie weiter von Fall zu Fall aus Vertretern der verschiedenen Branchen bestehen soll. Über das Ergebnis der Nürnberger Verhandlungen wird der Handelskammer und durch diese der bayerischen Regierung ein Bericht erstattet werden, und ich zweifle nicht, daf dieser Bericht auch uns im Hinblick auf unsere Mitwirkung durch mich vertraulich zugänglich gemacht wird.

Am Schluß meiner Ausführungen angelangt möchte ich nicht verfehlen, mich mit wenigen Worten noch mit dem Vorgehen der Tierärzte in neuerer Zeit zu befassen. Zeitungsberichten werden Sie über den Verlauf der letzten Tagung des Deutschen Veterinärrats schon Verschiedenes entnommen haben. Im Anschluß daran hat der Deutsche Veterinärrat im Juli 1921 in Ausführung eines Beschlusses seiner 17. Plenarversammlung, die in Weimar am 15. April 1921 stattfand, an Behörden usw. eine Denkschrift über die Notwendigkeit einer erweiterten Milchuntersuchung und über die Beteiligung der Tierärzte an der gesundheitlichen Überwachung des Verkehrs mit Milch übersandt. Diese Ausfübrungen des Deutschen Veterinärrats geben nach verschiedenen Richtungen durchaus fehl. Sie lassen zudem erkennen, daß der Veterinärrat mit den tatsächlichen Verbältnissen im Milchverkehr sowie mit der bisherigen Milchkontrolle nicht hinreichend vertraut ist. Seiner Eingabe liegt anscheinend das Bestreben zugrunde, den Tierärzten wegen der angeblichen Überfüllung ihres Standes ein neues Feld der Betätigung zu erschließen, und zwar ein Arbeitsgebiet, für das der Tierarzt weder zuständig noch schlechthin wissenschaftlich ausgebildet ist. Die Bedeutung des Tierarztes für die Versorgung der Bevölkerung mit einwandfreier Milch liegt auf dem Gebiete der Uberwachung des Gesundheitszustandes der Milchtiere. 
Zeitungsnachrichten zufolge ist bei den Verbandlungen des Veterinärrats am 15. A pril 1921 zum Ausdruck gekommen, daß leider auf diesem Gebiete wirksame gesetzliche Bestimmungen vorläufig nicht zu erreichen seien und daher der Tierarzt versuchen müsse, sich auf dem Gebiete der Überwachung des Verkehrs mit Milch zu betätigen. Hierin ist vom Standpunkte der öffentlichen Gesundheitspflege ein recht bedenklicher Fehlgriff zu erblicken. Denn der Tierarzt sollte dahin streben, durch die Überwachung des Milchviehs die Gewinnung einer gesundheitlich einwandfreien Milch zu gewährleisten. Sobald aber die Milch das Tier verlassen hat und als menschliches Nabrungsmittel in den Verkehr gebracht wird, unterliegt die Beaufsichtigung des Verkehrs mit Milch in wissenschaftlicher Hinsicht, soweit es sich um Verfälschungen, Nachahmungen, Verdorbenheit, Behandlung mit Fremdstoffen u. dergl. handelt, dem hierfür besonders ausgebildeten und geprüften Nahrungsmittelchemiker, soweit es sich jedoch um Fragen der Beschädigung des menschlichen Körpers durch Mikroorganismen und die durch solche verursachten Zersetzungsprodukte sowie um die Beurteilung der gesundheitlichen Wirkung von Zusätzen und Verunreinigungen aller Art handelt, dem für die Gesundheit des Menschen verantwortlichen Arzt, also nicht Tierarzt. Der Deutsche Veterinärrat hat zudem in ganz unbegreiflicher Weise vollständig verkannt, $\mathrm{da}$ die chemische Untersuchung der Milch bei Verfälschungen und Nachahmungen aller Art nicht lediglich aus wirtschaftlichen Gründen, also nicht deswegen erfolgt, weil der Verbraucher z. B. durch die Verabfolgung von verfälschter Milch mehr oder weniger an seinem Geldbeutel geschädigt wird, sondern vor allem deswegen, um zu verhüten, då der hilflose Säugling, der ausschließlich auf den Genuß von Milch angewiesen ist, unterernährt wird und daher in gesundheitlicher Hinsicht alle Folgen der Unterernährung zu tragen hat. Infolgedessen, aber nicht wegen des pekuniären Schadens allein, verwünscht die Mutter den Milchhändler, der ibr für ihr Kind verfälschte Milch liefert. Weiter ist verkannt worden, daß der gewissenhafte Nahrungsmittelchemiker z. B. beim Nachweis von Milchwässerung bestrebt ist, auch darüber Feststellungen zu treffen, ob das verwendete Wasser bereits als solches in gesundheitlicher Hinsicht höchst bedenklich, z. B. durch Zersetzungsprodukte menschlicher oder tierischer Abfallstoffe stark verunreinigt ist. Der Veterinärrat verkennt überhaupt den Umfang der Tätigkeit bei der Milchuntersuchung und scheint anzunehmen, daß selbst den Geruch, den Geschmack, den Schmutzgehalt und die Frische einer Milch nur der Tierarzt bedenkenfrei festzustellen vermöge. Hinsichtlich der Bedeutung der pathogenen Bakterien in der Milch übersieht der Veterinärrat vollständig die wirtschaftlichen Verhältnissen, mit denen der Praktiker naturgemäß rechnen muß. In einer Sammelmilch Krankheitskeime festzustellen, ist nicht schwierig; es ist auch bereits bekannt, in welchem Umfange derartige Keime im Milchverkehr vorkommen, aber unmöglich ist es, im allgemeinen, durch die Untersuchung der Sammelmilch festzustellen, von welcher Kuh die Infektion der Milch mit Krankheitserregern berrührt. Weiter ist zu beachten, daß jede verständige Hausfrau, bevor sie Milch verwendet, diese abzukochen pflegt, und daß alle im Verkehr mit Milch im allgemeinen in Betracht kommenden pathogenen Keime beim Abkochen vernichtet werden. Dies trifft z. B. auch hinsichtlich der Tuberkelbazillen zu, die im Hinblick auf den Umfang der Verbreitung der.Tuberkulose unter dem Milchvieh in der Sammelmilch in der Regel nachzuweisen sind. Weiter befremdet es auch, daß der Veterinärrat eine scharfe gesetzliche Abgrenzung der Befugnisse der Nahrungsmittelchemiker und Tierärzte hinsichtlich der Untersuchung von Milch verlangt. Denn letzten Endes liefert bekannt- 
lich der Sachverständige die zuverlässigsten Untersuchungsergebnisse, der auf einem Sondergebiete besonders ausgebildet ist, und bei den Chemikern liegt die Sache so, daß für die Untersuchung und Begutachtung der Lebensmittel Spezialisten herangebildet werden, die eine besondere Staatsprüfung, nämlich die der Nahrungsmittelchemiker, abzulegen haben. Der Tierarzt wird aber nicht etwa behaupten wollen, daß er sich neben seinen tierärztlichen Studien in der Nahrungsmittelchemie die Kenntnisse anzueignen vermöchte, die zu erwerben der Nahrungsmittelchemiker mindestens 9 - in der Regel einschließlich Promotion - 12 Semester braucht. Ein Blick auf die Prüfungsordnung der Nahrungsmittelchemiker läßt ohne weiteres ersehen, daß diese nicht lediglich als Chemiker, sondern auch auf dem Gebiete der Botanik, der Bakteriologie usw. ausgebildet sind. Trotzdem behauptet aber kein Nahrungsmittelchemiker, für die Beurteilung der Milch hinsichtlich der pathogenen Bakterien zuständig zu sein.

Grundsätzlich ist daran festzuhalten, daß für die Überwachung des Verkehrs mit Milch, soweit diese als menschliches Lebensmittel bestimmt ist, und zwar von dem Augenblick an, in dem die Milch das Tier verläßt, die Medizinalverwaltung zuständig ist, die sich bei, der Überwachung der Milch der öffentlichen Nahrungsmittel-Untersuchungsanstalten sowie der ärztlich geleiteten hygienischen Institute zu bedienen hat, und daß die Hygiene des Milchviehes in die Zuständigkeil des Tierarztes fällt.

Nebenbei sei noch bemerkt, daß Tierärzte in neuerer Zeit sogar die Forderung aufgestellt haben, für die Überwachung des gesamten Verkehrs mit solchen Lebensmitteln zuständig zu sein, die tierischen Ursprunges sind. Wie unhaltbar und wie unüberlegt derartige Forderungen. sind, ergibt sich z. B. schon daraus, daß eine eingehende Untersuchung der Fette auf chemischem Gebiete derartige Kenntniśs voraussetzt, die nur ein sehr erfahrener Chemiker haben kann, und daß es sich ähnlich mit der Chemie des Honigs usw. verhält. Selbst eine eingehende Untersuchung der Milch, die auch bei der Marktkontrolle jederzeit erforderlich wird, sobald abnorm beschaffene Milch vorliegt, erfordert die Kenntnisse eines gründlich ausgebildeten wissenschaftlichen Nahrungsmittelchemikers. Wie würde der Deutsche Veterinärrat darüber urteilen, wenn eines Tages die Deutschen Nahrungsmittelchemiker verlangen wollten, in der tierärztlichen Fleischbeschau weitgebend Verwendung zu finden? Aus den Kreisen der Nahrungsmittelchemiker sind derartige Forderungen bisher nicht gestellt worden, auch haben die Ärzte bisher nicht verlangt, daß ein Gesetz erlassen werde, das sich dahin ausspricht, daß nur sie für die Untersuchung der Milch auf Schmutzgehalt und Frische zuständig seien. In der Zeit der größten wirtschaftlichen Not, die wir durchmachen, darf aber gesetzlich eine mit sehr großen Kosten verbundene Kontrolle nur in dem Umfange und nach der Richtung durchgeführt werden, die im Interesse der Volksgesundheit zweifellos erforderlich ist. Standesinteressen haben hierbei auszuscheiden. Was würden schließlich unbefangen denkende Vertreter der Naturwissenschaften sagen, wenn die Botaniker - in Analogie zu den Tierärzten - verlangen wollten, alle die Lebensmittel, die letzten Endes pflanzlichen Ursprunges sind, also z. B. auch den Wein und die Liköre, zu untersuchen? Aber ich will davon absehen, satyrisch zu werden, da hierfür der Stoff und die Zeit zu ernst sind.

Indem ich hiermit schließe, empfehle ich Ihnen folgende Sätze zur Beschluß. fassung: 
1. Der Verein Deutscher Nahrungsmittelchemiker begrüßt im öffentlichen Interesse lebhaft die Bestrebungen des Herrn Reichsministers Dr. Hermes; er erklärt sich zugleich gern bereit, an der Lösung der vom Herrn Minister entwickelten Aufgaben tatkräftig mitzuarbeiten und hat daher den Wunsch, durch seinen Vorsitzenden in dem Beirat des Reichsernäbrungsministeriums zur Förderung ernährungswissenschaftlicher Forschungstätigkeit (jetzt „Reichsausschuß für Ernährungsforschung“) vertreten zu sein.

2. Der Verein wird, sobald die Regierung den Entwurf für ein neues Nahrungsmittelgesetz veröffentlicht, diesen Entwurf durch besondere, aus seinem Ausschuß gewählte Vertreter sorgfältig prüfen lassen, da in nächster Zeit eine Hauptversammlung nicht stattfinden kann, ganz abgesehen davon, daß ein großes Kollegium die mit der Prüfung des Entwurfes verbundene Kleinarbeit nicht gut erledigen kann.

3. Der Verein Deutscher Nahrungsmittelchemiker erhebt im öffentlichen Interesse Einspruch gegen die Denkschrift des Deutschen Veterinärrats vom Juli 1921 sowie gegen ähnliche Bestrebungen der Tierärzte, wobei er zugleich zum Ausdruck bringl, daß ihn hierbei keinesfalls etwa Standesinteressen leiten oder zu leiten brauchen, da die in Rede stehenden Bestrebungen der Tierärzte sachlich und wissenschaftlich der erforderlichen Grundlage entbehren.

\section{Anlage:}

Denkschrift über die Förderung ernährungswissenschaftlicher Forsehungstätigkeit durch das Reichsministerium füı Ernährung und Landwirtschaft.

(Als Erläuterung zu Kap. 1, Tit. 4 der einmaligen Ausgaben „Beihilfen für Forschungen auf dem Gebiete der Ernährung“.)

Vor dem Kriege ist das allgemeine Interesse für wissenschaftliche Fragen auf dem Gebiete der Frnährung des Menschen verbältnismäßıg gering gewesen. Der vornehmlichste Grund hierfür lag wohl in der Tatsache, daf ein Mangel an Lebensmitteln nicht bestand und die wirtschaftlichen Verhältnisse nicht so zu unbedingter Sparsamkeit drängten wie jetzt. Trotzdem hat in Deutschland die im wesentlichen auf privater Initiative fußsende Ernährungswissenschaft ungewöhnliche Erfolge erzielt, die sich alsbald anch befruchtend für die praktische Anwendung erwiesen baben. Die Landwirtschaft ist aus ihrem Jahrtausende alten rein empirischen Verfahren zur rationellen Wirtschaft übergegangen, hat die Bodenerträgnisse gesichert und vervielfacht. Nicbt minder erfolgreich ist die Einführung rationeller Fütterungsmethoden für die Viehzucht geworden, auch in der Fischzucht könnten die Erträgnisse erstaunlich gesteigert werden. Die Vorteile des "rationellen" Verfahrens liegen hier überall auf der Hand.

Dagegen lagen die Erfolge der Ernährungswissenschaft hinsichtlich der Ernährung des Menschen mehr aut medizinischem Gebiete (Durchführung der diätetischen Therapie, Studium der Sänglingsernährung u. a. m.), wobei insbesondere auch des erfolgreichen Schutzes gedacht werden soll, den der staat auf Grund der Ergebnisse der Ernährungswissenschaft gegenüber den Gefahren durch verdorbene, verfälschte, nachgemachte und gesundheitsschädliche Nahrungsmittel gewähren konnte.

Das Studium des eigentlichen Problems der Volksernährung ist erst kurz vor Beginn des Krieges von der Wissenschaft begonnen worden. Die wissenschaftlichen Leistungen auf diesem Gebiete fußen vorwiegend auf dem freien Entschluß einzelner Gelehrter. Im Gegensatz hierzu ist in auferdeutschen Ländern das Interesse für Ernährungswissenschaft, und zwar sowohl für das Studium der Nahrungsmittel wie für das Studium der ernährungswissenschaftlichen Probleme überhaupt auferordentlich großs. Diesem lebhaften Interesse tür die Erforsehung ernährungswissenschaftlicher Fragen verdanken u. a. die Carnegie Institution of nutrition in Boston, eine der grofartigsten Stiftungen der Welt, sowie zahlreiche andere vorzüglich aus: gestattete Speziallaboratorien in Amerika ihre Entstehnng. Auch Holland wird im kommenden Jahre ein „Institat für Volksernährung“ erhalten, und selbst die 'T'schecho-Slowakei plant auf Initiative ihres Präsidenten die Erriehtung eines derartigen Instituts.

So dringend notwendig die Not der Zeit die systematische Erforschung des Problems der Volksernährung erseheinen läkst, so gestattet doch die finanzielle Bedrängnis dem Reiche nicht, den Weg, den außerdeutsche Staaten zur Erreichung dieses Zieles gehen, ebenfalls zu beschreiten und dem Reichsministerium für Ernährung and Land wirtschaft besondere technische 
Reichsinstitute für die auf dem Gebiete der Ernährungswissenschaft za lösenden Aufgaben zur Verfügung za stellen. Das Reichsministerium für Ernährung und Landwirtschaft hat diesem Umstande Rechnung getragen, es aber in Anbetracht der Bedeutung, die das Problem der Volksernährung für das Volkswohl hat, für seine Pflıcht gehalten. anregend und fördernd auf die deutsche Wissenschaft dadurch einzuwirken, daf es dem Ministerium einen „Reichsansschuß für Ernährungsforschung“ angegliedert hat. Aufyabe dieses Ausschusses soll es in erster Linie sein, eine enge Verbindung zwischen den Vertretern der Ernährungswissenschalt und dem Ministerium herzustellen, damit es dem Ministerium ermöglicht wird, die Aufmerksamkeit der Wissenschaft auf besonders dringliche, vom Standpunkte der Verwaltung interessierende Fragen der Volksernährung zu lenken. Daneben soll der Ausschußs bei seinen Beratungen in wissenschaftlicher; technischer und volkswirtschaftlicher Hinsicht zagleich fortgesetzt anregend auf seine eigenen Mitglieder wirken. durch den gemeinsamen Gedankenaustauseh von Vertretern aller beteiligten naturwissenschaftlichen Kreise eine Zersplitterung der Kräfte vermeiden und damit zur vollen Entwickelung aller für die Volksernährung in Betracht kommenden Kräfte beitragen.

In den Ausschuf sind bisher berafen worden die Professoren Dr. Emil Abderhalden in Halle a. S.; Dr. Baur in Potsdam; Dr. Ernst Beckmann in Berlin.Dahlem; Dr. Gottlieb Haberlandt in Berlin-Dahlem; Dr. A. Heiduschka in Dresden; Dr. Karl A. Hofmann in Charlottenburg; Dr. A. Juckenack in Berlin; Dr. O. L emmermann in Berlin; Dr. med. Friedrich von Müller in München; Dr. R. O. Neumann in Bonn; Dr. Th. Paul in München; Dr. M. Rubner in Berlin; Dr. R. Willstätter in München and der Statistiker Dr. René Kuczynski in Zehlendorf. Das Reichsgesundbeitsamt wird zu den Sitzungen des Ausschusses eingeladen.

Mit Hilfe des Reichsausschusses für Ernährungsforschung und der ihm zar Verfügung zu stellenden Mittel will das Reichsministerium für Ernährung und Landwirtschaft die deutsche Ernährungswissenschaft unterstützen und anregen zur Erforschun $₫$ von Fragen nach Vermehrang der Quellen der Nahrungsyewinnang, nach sachgemäßer Aufbewahrung (Konservierung) der gewonnenen Rohstoffe und nach der vorteilhaftesten Art der Verwendung und Ausnutzung dieser Rohstoffe für die menschliche Ernährung unter besonderer Beachtung ernährungsphysiologischer Gesichtspunkte.

Dabei ist es der ausgesprocbene Zweck des Reichsministeriums für Ernährung und Landwirtschaft, die Wissenschaft für Fragen zu interessieren, deren Ergebnisse volkswirtschaftlich von Bedeutung sind. Unter den zahlreichen Fragen, die nach dieser Richtung hin noch ungeklärt sind, seien nachstehend folgende aufgeführt:

1. Das Brot. Von den bei der Ausnutzung des Getreides zum Zwecke der Herstellung von Brot wissenschaftlich interessierenden Fragen steht in erster Linie die Frage, ob und wie es möglich ist, sämtliche Nährstoffe des Getreidekornes dem unmittelbaren menschlichen Genuf zugänglich zu machen, sodaß sie weitestgehend ausgenutzt werden können und das Brot zugleich ein leicht verdauliches and angenehm schmerkendes Lebensmittel darstellt. Fis ist bisher noch keineswegs in befriedigender Weise geglückt, den nährstoffreichen Inhalt gewisser wichtiger Kleiezellen den Verdauungssäften des menschlichen Körpers zagängig zu machen, und auch die Verbesserung der Herstellungsart des Brotes bedarf noch eingehendsten Studiums.

2. Die Fette. Die wissenschaftlichen Kenntnisse über die Fette sind noch verhältnismäfig gering, und zwar sowohl was die qualitative Zusammensetzung der Speisefette betrifft, wie namentlich auch hinsichtlich der quantitativen Beschaffenheit der Stoffe. Zunächst muß hier die Forschung intensiver einsetzen, damit klarer gesehen werden kann. Im übrigen versucht die Wissenschaft seit einiger Zeit auf chemischem Wege aus Kohlenwasserstoffen, den leicht zugänglichen Anteilen des Braunkohlenteers, Fettsäuren herzustellen. Es ist nun zu untersuchen, inwieweit diese, zunächst für technische Zwecke (wie z. B. Seifenfabrikation) bedeutsamen Produkte auch für die Ernährung herangezogen werden können. Auch die Frage der Verwertung anderer Fettsäureester (wie z. B. Athyl- oder Glycolester) an Stelle von Glycerinesten, aus denen in der Hauptsache die Fette bestehen, ist physiologiseh eingehend zu prüfen; vielleicht gelingt es auf diese Weise, für die höheren Fettsäuren, wie Stearinsäure eine bessere Ausnutzungsmöglichkeit zu schaffen.

3. Das Eiweif. Noch komplizierter als das Gebiet der Fettchemie ist das Gebiet der Kiweißchemie, um das sich in der letzten Zeit seines Lebens insbesondere der berühmte Chemiker Fmil Fischer ganz hervorragende Verdienste erworben hatte. Trotzdem gibt es aber auf diesem Gebiete noch eine Reihe von Fragen zu lösen, die vom ernährungswissensehaftlichen Standpunkte von weittıagender Bedeutung sein können.

4. Der Frage der Deckung des Fettbedarfes der Bevölkerung sollen ferner Versuche dienen, die mit verschiedenen Sorten der Sojabohnen in Deutschland angestellt worden sind, und deren Förderang und Fortsetzung im Hinblick auf den großen Fett- und Eiweißgehalt der Sojabohne auferordentlich erwïnscht ist. Daneben interessiert z. B. auch der Versuch des Anbaues einer anderen, ein gutes Speiseöl liefernden Pflanze, nämlich der Erdnús. 
5. Den Zucker. Es ist bekannt, daB während des Krieges versucht wurde, Holz (Saggespäne) zu verzuckern, um auf diese Weise Alkohol ohne Verarbeirung von Nahrungsund Futtermitteln herzustellen. Die angewendeten Verfabren waren lediglich auf die Alkoholgewinnung und die Kriegswirtschaft zugeschnitten. Andererseits berechtigen aber andere Arbeiten über die Verzuckerung der Cellulose des Holzes zu der Annahme, dâ es gelingen kamn, Holz, insbesondere Holzabfalle, sehr weitgehend für verschiedono $Z$ wecke in Zucker uberzuführen.

6. Selbst die Klauen der Schlachttiere und ähnliche Abfalle von hornartigen Stoffen sind bereits chemiseh in Körper ubergefuhrt worden, die - sei es direkt, sei es indirekt - einmal in der Ernahrungswirtschaft von Bedeutung sein können.

7. Zahlreich sind endlich die Wragen, die auf ernahrungs-physiologischem Gebiete noch ungeklät sind, und deren Erforschung für die Volksernährung von weitestgehender Bedeutung werden kann. So bedarf z. B. die Frage, welche Rolle die anorganischen Verbindungen (Mineralstoffe bezw. sog. Nährsalze) im Leben des Menschen, des Nutztieres, der Nutzpflanzen usw. spielen, noch eines sehr gründlichen Studiums. Allgemein bekannt ist es ferner seit mehreren Jahren, $d a \mathcal{B}$ es gewisse Ergänzangsstoffe der Ernährung, Vitamine, gibt, deren Fehlen in der Nahrung zu schweren Ernährungsstörungen und damit Krankheiten führen kann. Über die zur Zeit noch dunkle Natur dieser Körper muß versucht werden, eingehenden Aufschluß zu gewinnen. Ebenso dunkel ist zum Teil noch das Gebiet der Enzyme, jener Stuffe, die im Leben des Menschen, Tieres und der Pflanze eine außerordentlich große Rolle spielen.

In der am 4. Juli 1921 abgehaltenen 1. Sitzung des Ausschusses ist ein vorläufiges Arbeitsprogramm entworfen worden. Die Mitglieder des Ausschusses haben den Bestrebungen des Reichsministeriums für Ernährung und Landwirtschaft lebnaftes Interesse entgegengebracht und es gern übernommen. an die Bearbeitang einschlägiger Fragen heranzutreten. Zur Durchführung dieser Arbeiten hat das Reichsministerium für Eirnährung und Landwirtschaft aus den in Kap. 1, Tit. 4 der einmaligen Ausgaben des Haushaits $1920 / 21$ ihm zur Verfugung stehenden Mitteln Beihilfen an einzelne Forscher gewährt. Diese Arbeiten bewegen sich im wesentlichen in dem oben angezogenen Rahmen, berücksichtigen dabei aber auch ernährungsstatistische Hrhebangen. Es hat sich jedoch herausgestellt, daßs die dem Reichsministerium für Ernährung und Landwirtschaft zur Verfügung stehende Summe, um amfancreiche Versuche in Angriff nehmen za können, bei der fortschreitenden Entwertung des Geldes viel zu niedrig ist. Die Kosten für die Bezahlung wissenschaftlicher Hilfskräfte und für die Durchführung wissenschaftlicher Versuche (Gas, eloktrischer Strom, Untersuchungsmaterialien u. a.) haben sich seit vorigem Jahre etwa verdoppelt. Aus diesem Grunde ist eine Erhöhung des Fonds auf 500000 M. unerläßlich.

Die Aufwendung dieser Mittel wird sich zweifellos auch volkswirtschaftlich lohnen. Welche Lrfolge zielbewafte Forschungstätixkeit auf dem großen Gebiete der Ernahrang im weitesten Sinne haben kann, beweisen die Unwäluangen, die nach den eingangs erwahnten wissenschafthichen Erfolgen auf dem Gebiete der Ernahrung der Pflanzen und des Viehes einyetreten sind, beweist aber vor allem auch das Beispiel der künstichen Stickstofferzengung, die der deutschen Volkswirtschaft zu unermeklichem Segen gereichen kann.

Vorsitzender: Ich stelle den Vortrag des Herrn Geheimrat Juckenack zur Diskussion. Vorerst will ich bemerken, daß im Interesse einer ordnungsgemäßigen Protokollierung es notwendig ist, dal jeder Diskussionsredner vor Beginn seiner Ausführungen deutlich seinen Namen und seinen Wobnort angibt. Ich frage nun, wer das Wort zu den Ausführungen des Herrn Geheimrat Juckenack wünscht. Es scheint das Wort nicht verlangt zu werden, und damit erkenne ich Ihre Zustimmung zu den Ausführungen des Herrn Geheimrat Juckenack und ebenso auch zu den Anträgen, in welche er seine Ausführungen am Schlusse zusammengefaßt hat. Danach würde einmal der Verein an den Herrn Ernährungsminister Dr. Hermes die Bitte richten, den Vorsitzenden des Vereins zum Mitglied des Beirats des Reichsernährungsministeriums zu ernennen, zweitens würde heute eine Konmission zu ernennen sein, welche den demnächst der Öffentlichkeit vermutlich übermittelte Entwurf eines neuen Nabrungsmittelgesetzes prüfen und die Ausführungen den zuständigen Reichsbehörden zu übermitteln bätte. Ich möchte zunächst Vorschläge von Ihnen hören, wie dieser Ausschuß zur Prüfung des Entwurfs eines neuen Nahrungsmittelgesetzes zusammengesetzt sein soll. 
Geheimrat Juckenack: Ich schlage vor, aus dem Kreis der Ausschußmitglieder und deren Vertreter durch den Vorstand die Kommission zu wählen. Es müssen Herren sein, die in der praktischen Lebensmittelkontrolle tätig sind, und zwar teils im Staatsdienst, teils im Kommunaldienst. Am besten überläßt man dem Vorstand die Auswahl der Herren. (Zustimmung.)

Vorsitzender: Werden die Herren einverstanden sein, daß der engere Vorstand aus den Ausschußmitgliedern und deren Stellvertretern diesen Ausschub zusammensetzt? (Zustimmung!) Dann bin ich noch verpflichtet, Thnen, Kollege Juckenack, noch aufrichtigen Dank zu sagen für den inhaltreichen und interessanten Vortrag, mit dem Sie alle diejenigen Fragen beleuchtet haben, die für uns als Nahrungsmittelchemiker von besonderem Interesse sind. Wir hoffen und erwarten, daß Ihre Ausführungen für unsere demnächstigen Arbeiten in den kommenden Jahren von segensreichem Einfluß sein werden.

Wir gehen nunmehr in unserer Tagesordnung weiter, und ich erteile Herrn Prof. Dr. Behre das Wort zu seinem Vortrag:

\title{
Die Methoden der Kunsthoniguntersuchung, insbesondere der Bestimmung der Saccharose und des Stärkesirups.
}

\author{
Von \\ Prof. Dr. A. Behre, \\ Direktor des Chemischen Untersuchungsamtes der Stadt Chemnitz.
}

(Nach z. T. in Gemeinschaft mit A. Düring und H. Ehrecke ausgeführten Untersuchungen.)

M. H.! Auf der Tagesordnung unserer diesjährigen Versammlung steht als Gegenstand die Beratung und endgültige Beschluffassung über Bestimmungen, die uns Richtlinien für den Handelsverkehr mit Kunsthonig geben sollen. Diese Richtlinien enthalten auch Angaben über den Mindestgehalt an Trockensubstanz, den Höchstgehalt an Saccharose, Stärkesirup, Asche, Säure usw. Ohne Ihren am morgigen Tage zu fassenden Beschlüssen vorgreifen zu wollen, möchte ich es unternehmen, unsere Beratungen vorbereitend, vor Ihnen die Verfahren der Bestimmung einzelner Bestandteile des Kunsthonigs und ihre Brauchbarkeit zu erörtern; denn daß wir, wenn wir derartige Beschlüsse von weitreichender Tragweite für die Industrie fassen, auch Mittel zum Nachweis der Bestandteile oder Fälschungsmittel besitzen müssen, ist selbstverständliche Voraussetzung und kann von der beteiligten Industrie verlangt werden. Bei den Beratungen des Ausschusses für Kunsthonig ist seitens einiger Vertreter der Kunsthonigindustrie der Zweifel ausgesprochen worden, ob es der analytischen Chemie gelingen würde, den Gehalt eines Kunsthonigs an Saccharose neben Stärkesirup, falls letzterer zugelassen werden würde, mit hinreichender Genauigkeit festzustellen. Seitens der Chemiker des Ausschusses glaubte dieses bejaht werden zu können. Den Beweis dafür, dafo dieses mit Recht geschah, möchte ich Ihnen heute erbringen, obgleich ich bekennen muß, daß wir nicht alle völlig überzeugt davon waren, daß die bisher angewendeten Bestimmungsverfahren völlig befriedigende Ergebnisse liefern würden. Gerade diese Unsicherheit hat mich angeregt, meine bereits vor mehreren Jahren begonnenen Untersuchungen über die Zusammensetzung des Kunsthonigs weiterzuführen.

Ich beabsichtige nicht, die Verfahren der Bestimmung aller Bestandteile des Kunsthonigs hier in die Erörterung zu ziehen, z. B. sind die Bestimmungsverfahren 